\title{
G

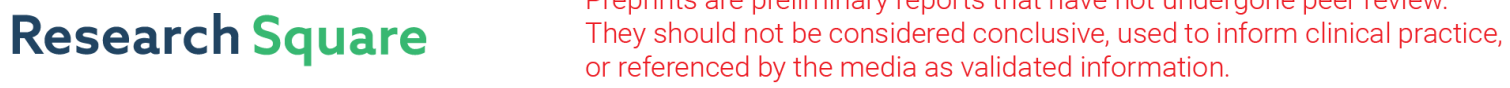 \\ Key Residues of Bacillus Thuringiensis Cry2Ab for Oligomerization and Pore-Formation Activity
}

\section{Zhi-Zhen Pan}

Fujian Academy of Agriculture Sciences: Fujian Academy of Agricultural Sciences

\section{Lian Xu}

Xiamen University School of Life Sciences

\section{Bo Liu}

Fujian Academy of Agriculture Sciences: Fujian Academy of Agricultural Sciences

\section{Qing-Xi Chen}

Xiamen University School of Life Sciences

\section{Yu-Jing Zhu ( zyjingfz@163.com )}

Fujian Academy of Agricultural Sciences https://orcid.org/0000-0002-0045-6708

\section{Original article}

Keywords: Cry2Ab, oligomerization, insecticidal activity, pore-forming activity.

Posted Date: May 13th, 2021

DOI: https://doi.org/10.21203/rs.3.rs-510564/v1

License: (c) (1) This work is licensed under a Creative Commons Attribution 4.0 International License. Read Full License

Version of Record: A version of this preprint was published at AMB Express on July 31st, 2021. See the published version at https://doi.org/10.1186/s13568-021-01270-0. 


\section{Abstract}

As a pore-forming toxin, oligomerization and pore-formation were both required for the mode of action of Cry toxins. Previous studies revealed that Domain I helices a4-a5 were involved in oligomerization of Cry2Ab, while the active residues in charge of Cry2Ab aggregation remained ambiguous. In present studies, we built 20 Cry2Ab alanine mutants site directed in helices a4-a5 and demonstrated that mutants N151A, T152A, F157A, L183A, L185A and I188A blocked the assembly of $250 \mathrm{kDa}$ oligomers, suggesting that those residues were key residues for Cry2Ab oligomerization. As expected, those variants severely reduced the insecticidal activity against $P$. xylostella which was similar to our previous reports. Furthermore, we found that the pore-forming activities of non-oligomerization mutants sharply decreased compared to wild-type Cry2Ab. Taken together, our data comprehensively identified key residues for Cry2Ab for the first time and emphasized that oligomerization was closely related to insecticidal activity and pore-forming activity in Cry2Ab.

\section{Introduction}

Cry toxin, an insecticidal crystal toxin derived from Bacillus thuringiensis (Bt), is widely applied as a bioinsecticides to control agricultural pests all over the world (Brave et al. 2011; Schnepf et al. 1998). Cry toxins are produced in an inactive form called protoxin and proteolytic activated by insect midgut protease. Activated Cry toxins interacted with midgut receptors in target insects, self-aggregated to form pre-pore oligomeric structures and inserted into the cell membrane to form pores, resulting in the death of insects (Pardo-Lopez et al. 2013; Bravo et al. 2007; Vachon et al. 2012).

At present, most researches have been devoted into "interaction of Cry toxins with midgut receptors" since which is an essential step for the insecticidal mechanism of Cry toxins (Pigott et al. 2007; Bravo et al. 2013). A number of insect midgut proteins such as cadherin, aminopeptidase-N (APN), alkaline phosphatase (ALP) and ATP-binding cassette transporter subfamily $\mathrm{C}$ member 2 (ABCC2) had been proposed as functional receptors for Cry toxins (Pigott et al. 2007; Zhou et al. 2017; Park et al. 2009; Guo et al. 2015; Chen et al. 2017). It was suggested that the mutation or down-regulation of Cry receptor genes were tightly linked with high level of Cry resistance in diverse insects (Ferré et al. 2002; Baxter et al. 2011). In most cases, Cry toxins were produced in an inactive protoxin and the proteolytic activation was required for insecticidal activity of Cry toxins (Xu et al. 2016). It was also reported that improper processing of Cry toxins was associated with Cry resistance in lepidopteran insects (Liu et al. 2014; Xia et al. 2016). Those studies made a closer look on the mechanism of Cry toxins and provided new strategies for agricultural pests control.

As a pore-forming toxin, Cry toxins kill insects by forming pore on their midgut cells. Assembly of pre-pore oligomers was required for pore-forming processing of Cry toxins (Jiménez-Juárez et al. 2013; Vachon et al. 2002). Domain I, a seven-helix bundle, was wildly reported involved in the assembly of pre-pore structure and membrane channel formation (Pardo-Lopez et al. 2013; Vachon et al. 2012). It was proposed that a binding step of cadherin receptors and proteolytic removal of helix a-1 were required for 
oligomerization of Cry1A toxin (Soberón et al. 2007). Helix a-3 was found to participate in toxin oligomerization as the mutation on this region affected oligomerization, as well as toxicity of Cry toxin (Vachon et al. 2002; Muñoz-Garay et al. 2009). Recently, Pacheco et al. reported that an intramolecular salt bridge in helix a-3 was essential for the stability and oligomerization of Cry4Ba toxins (Pacheco et al. 2017). The helices $a-4$ and $a-5$ had been wildly reported as a pore-forming region, as they were the only helices capable of adopting a transmembrane orientation (Girard et al. 2008; Torres et al. 2008). An umbrella model was further proposed in which the helices $a-4$ and a-5 inserted into the membrane to form pores while other a-helices covered the surface of membrane (Pardo-Lopez et al. 2013).

Our previous study suggested that the exposure of helices a4-a5 was required for oligomerization and insecticidal activity of Cry2Ab (Xu et al. 2018). However, the key residues involved in the oligomerization activity of Cry2Ab were still unknown. In the present study, we sought to determine the key residues of Cry2Ab for the oligomerization activity by constructing 20 alanine mutants site directed on helices a4-a5. It revealed that residues N151, T152, F157, L183, L185 and I188 are involved in Cry2Ab oligomerization. The non-aggregation Cry2Ab mutants not only affected the insecticidal activities against Plutella xylostella, but also weakened the pore-forming activities on liposome. Our data firstly identified key residues of Cry2Ab for oligomerization activity and highlighted that oligomerization was tightly linked with the pore-forming activity of Cry2Ab.

\section{Materials And Methods}

\section{Insects}

A laboratory population of $P$. xylostella larvae was purchased from Henan Jiyuan Baiyun Industry Co., $L t d$, China. $P$. xylostella larvae were fed with an artificial diet and maintained under environmental conditions of $27 \pm 2{ }^{\circ} \mathrm{C}, 70 \%$ of humidity, and photoperiod of $14: 10 \mathrm{~h}$ (light/dark).

\section{Construction of Cry2Ab variants}

Twenty Cry2Ab mutants site-directed on helices a4-a5 in Domain I were built by replacing V150, N151, T152, M153, Q154, Q155, L156, F157, L158, N159, R160, N182, L183, H184, L185, S186, F187, I188, R189, D190 with alanine. The Cry2Ab mutants were obtained by overlapping-extension PCR using wild-type Cry2Ab DNA fragment as template ( $X u$ et al. 2018). Primers used for generation of site-directed mutagenesis in PCR fragments were listed in Supplementary Table S1. The mutated cry2Ab fragments were further digested by the restriction enzymes $B g / I I$ and $E c o R I$, which were then ligated into the plasmid pET30a. Recombined plasmids were transformed into Escherichia coli BL21 (DE3) cells and positive clones were verified by PCR, restriction enzyme digestion and DNA sequencing (Xu et al. 2018).

\section{Construction of Cry2Ab variants}

The production and purification of wild-type and mutant Cry2Ab toxins were performed as previously described (Pan et al. 2014). E. coli BL21 (DE3) cells harbouring pET30a-cry2Ab were grown in LB medium 
containing $35 \mu \mathrm{g} / \mathrm{mL}$ kanamycin. The expression of wild-type and mutants Cry2Ab protoxins were induced overnight at $25^{\circ} \mathrm{C}$ with $0.5 \mathrm{mM}$ isopropyl-B-D-thiogalactopyranoside (IPTG) after $\mathrm{OD}_{600 \mathrm{~nm}}$ reached 0.4-0.6. Cry2Ab toxins were purified using a Ni-IDA Prepacked Column (Sangon, CHINA). Purified Cry2Ab were detected by $10 \%$ SDS-PAGE and western blotting using an anti-Cry2Ab antibody. To generate Cry2Ab activated-toxin, Cry2Ab protoxin was incubated with $P$. xylostella midgut juice (PxMJ) at $30^{\circ} \mathrm{C}$ for 60 minutes at a mass ratio of 20:1 (m: m). Protein concentration of Cry2Ab toxins were determined using a BCA Protein Assay Kit (Beyotime, China).

\section{Oligomerization assay}

For oligomeric formation assays, wild-type and mutants Cry2Ab toxins were activated by PxMJ in the present of PxBBMV and exchanged into sodium carbonate buffer $(50 \mathrm{mM}, \mathrm{pH}$ 9.5) using a PD-10 desalting column. The aggregation of Cry2Ab was processed at $30^{\circ} \mathrm{C}$ overnight. The Cry2Ab proteins were mixed with $5 \times$ SDS-PAGE loading buffer and incubated at $60^{\circ} \mathrm{C}$ for $10 \mathrm{~min}$. The oligomeric formation of Cry2Ab was detected by $8 \%$ SDS-PAGE with Coomassie staining (Xu et al. 2018).

\section{Bioassay}

Bioassays of wild-type and mutants Cry2Ab toxins against second instar larvae of $P$. xylostella were estimated according to Pan et al. (Pan et al. 2014). Five or six concentrations of Cry2Ab toxins were set up and sodium carbonate buffer $(50 \mathrm{mM}, \mathrm{pH}$ 9.5) was served as a negative control. Thirty second instar larvae of $P$. xylostella were used for each concentration assay and three independent replicates were performed. The median lethal concentration $\left(\mathrm{LC}_{50}\right)$ value were analyzed by SPSS 17.0 (Statistical Product and Service Solutions) using PROBIT analysis (Finney, 1971) (Finney et al. 1971). The relative potency (\%) had been normalized to the $\mathrm{LC}_{50}$ value of wild-type Cry2Ab.

\section{Liposome leakage assay}

Liposome preparation was performed as described by Ding et al. (Ding et al. 2016). Phosphatidylcholine, phosphatidylethanolamine and cholesterol were dissolved in chloroform and mixed in a 4:4:2 proportion (molar mass). The mixed lipids were put in a glass vial and evaporated under a stream of nitrogen to form lipid film. The lipid film was then shaking with SUV-1 buffer ( $20 \mathrm{mM}$ HEPES, $50 \mathrm{mM} \mathrm{NaCl}, 3 \mathrm{mM}$ calcein, $\mathrm{pH}$ 7.5) at room temperature for 2 hours. Liposomes were generated by extrusion of the hydrated lipids through a 100-nm polycarbonate filter (Whatman) 35 times using a Mini-Extruder device (Avanti Polar Lipids Inc). Calcein, outside the liposome, were removed by exchanging the liposome into SUV-2 buffer (20 mM HEPES, $50 \mathrm{mM} \mathrm{NaCl}$, pH 7.5) using a Sephadex G-50 column. Liposomes were stored at 4 ${ }^{\circ} \mathrm{C}$ and used within $48 \mathrm{~h}$.

For liposome leakage assay, the liposome encapsulated calcein was diluted to $200 \mu \mathrm{M}$ in SUV-2 buffer supplemented with $3 \mu \mathrm{M} \mathrm{MnCl}_{2}$. The released calcein could be quenched by $\mathrm{MnCl}_{2}$ in the solution. The excitation and emission wavelengths were set as $490 \mathrm{~nm}$ and $520 \mathrm{~nm}$, respectively, to examine the fluorescence of calcein. $480 \mu \mathrm{L}$ of liposome was added to the cuvette and the emission fluorescence was 
readed as $\mathrm{F}_{\mathrm{t} 0} .20 \mu \mathrm{L}$ of Cry2Ab was then added to and the emission fluorescence was continuously recorded as Ft at 10 s intervals. After $10 \mathrm{~min}, 20 \mu \mathrm{L}$ of $10 \%$ Triton X-100 was added to achieve complete release of calcein and the fluorescence records were defined as $F_{t 100}$. The percentage of liposome leakage at each time point is defined as: leakage $(t)(\%)=\left(F_{t}-F_{t 0}\right) \times 100 /\left(F_{t 100}-F_{t 0}\right)$.

\section{Results}

\section{Construction of Cry2Ab mutants sited-directed on helices a4-a5}

Our previous studies demonstrated that helices a4-a5 in Domain I was involved in oligomerization of Cry2Ab since some Cry2Ab mutants (TM152153AA, LF156157AA, NR159160AA, LH183184AA, Fl187188AA) failed to assemble $250 \mathrm{kDa}$ oligomers (Fig 1A) (Xu et al., 2018). Those results suggested that the active regions for Cry2Ab oligomerization were limited to $V^{150}-R^{160}$ in helix $a-4$ and $N^{182}-D^{190}$ in helix a-5 (Fig. 1B). To further authenticate single residues for Cry2Ab oligomerization, 11 Cry2Ab mutants site-directed on helix a-4 (V150A, N151A, T152A, M153A, Q154A, Q155A, L156A, F157A, L158A, N159A, R160A) and 9 Cry2Ab mutants site-directed on helix a-5区N182A, L183A, H184A, L185A, S186A, F187A, I188A, R189A, D190A囚were constructed using Escherichia coli expression system.

The upstream and downstream DNA fragments of Cry2Ab mutants contained mutation sites were amplified by PCR (Fig. S1A and S1B) and the full-length of DNA fragments of Cry2Ab mutants were obtained by overlap extension PCR (Fig. S1C). The Cry2Ab DNA was ligated to plasmid pET30a and transformation into BL21 competent cells. The recombinant plasmids were verified by PCR using T7 primers and a DNA product about 2300 bp could be amplified (Fig. S2). The recombinant plasmids were further digested with $B g / I I$ and $E c O R I$ and a pET30a fragment (about $5500 \mathrm{bp}$ ) and a Cry2Ab fragment (about 1900 bp) could be detected (Fig. S3). Those results suggested that the Cry2Ab DNA was ligated into PET30a. DNA sequencing revealed that the specific sites of $20 \mathrm{Cry} 2 \mathrm{Ab}$ mutants had been replaced by alanine.

\section{Production and identification of Cry2Ab mutants}

The expression of Cry2Ab mutants were performed under the induction of $0.5 \mathrm{mM}$ isopropyl- $\beta$-dthiogalactoside. SDS-PAGE revealed that all Cry2Ab mutants could be induced-produced with a molecular weight of $65 \mathrm{kDa}$ (Fig. 2A). Those Cry2Ab mutants further purified by a Ni-IDA prepacked column (Fig. 2B) and could be detected by an anti-Cry2Ab antibody (Fig. 2C). Furthermore, proteolysis assay indicated that all Cry2Ab variants could be processed into $50 \mathrm{kDa}$ activated-toxins by PxMJ, which were similar to wildtype Cry2Ab (Fig. 2D). These results revealed that mutations in the helices a4-a5 did not cause a major structural disturbance in Cry2Ab.

\section{Authentication of key residues for Cry2Ab oligomerization}

The assembly of $250 \mathrm{kDa}$ oligomer of variants Cry2Ab were evaluated by $8 \%$ SDS-PAGE (Fig. 3A). Wildtype Cry2Ab could form 250 kDa oligomers which was similar to our previous reports (Xu et al. 2018). Six 
Cry2Ab mutants (N151A, T152A, F157A in helix a-4 and L183A, L185A, I188A in helix a-5) failed to assemble $250 \mathrm{kDa}$ oligomers (defined as non-oligomerization group). Eight Cry2Ab mutants (M153A, Q154A, L156A, N159A, R160A in helix a-4 and N182A, H184A, R189A in helix a-5) greatly reduced the assembly of $250 \mathrm{kDa}$ oligomers (defined as reduced oligomerization group). The remaining Cry2Ab variants (V150A, Q155A, L158A in helix a-4 and S186A, F187A, D190A in helix a-5) could aggregate and form 250 kDa pre-pore structure (defined as normal oligomerization group) which were similar to wildtype Cry2Ab.

To further assess the oligomers formation of Cry2Ab, we employed Image $\mathrm{J}$ software to evaluate the percentage of oligomer (oligomer / monomer $\times 100 \%$ ). As shown in Fig 3B, the proportion of oligomer and monomer in normal oligomerization group was about 80\%-110\%, which was similar to wild-type Cry2Ab (about 95\%). However, this value in reduced oligomerization group was about 40-60\% and in nonaggregation group were less than $20 \%$. Those results suggested that residues N151, T152, F157, L183, L185, I188 might serve as key residues for Cry2Ab oligomerization.

\section{Oligomerization is associated with insecticidal activity of Cry2Ab}

We further assessed the insecticidal activities of non-oligomerization group (N151A, T152A, F157A, L183A, L185A and I188A) Cry2Ab mutants against second instar larvae of $P$. xylostella, with wild-type Cry2Ab, V150A and S186A (two Cry2Ab mutants in normal oligomerization group) as positive control (Figure 4). The $L_{50}$ values of $\mathrm{V} 150 \mathrm{~A}$ and S186A was 1.978 and $1.432 \mu \mathrm{g} / \mathrm{cm}^{2}$, which were close to that of wild-type Cry2Ab $\left(1.458 \mu \mathrm{g} / \mathrm{cm}^{2}\right)$. However, the LC $_{50}$ values of N151A, T152A, F157A, L183A, L185A and I188A were 5.097, 4.232, 3.234, 3.083, 3.579 and $3.545 \mu \mathrm{g} / \mathrm{cm}^{2}$, respectively, all of which were higher than that of wild-type Cry2Ab $\left(1.458 \mu \mathrm{g} / \mathrm{cm}^{2}\right)$.

\section{Oligomerization is associated with pore-forming activity of Cry2Ab}

The pore-forming activities of non-oligomerization group (N151A, T152A, F157A, L183A, L185A and I188A) Cry2Ab mutants were further evaluated by time course of liposome leakage assay. As shown in Figure 5, wild-type Cry2Ab could make pore on liposome and led to high leakage of calcein into solution, which was the same with V150A and S186A. The maximum calcein release percentage caused by wildtype Cry2Ab, V150A and S186A were 60.95\%, 55.87\%, and 58.73\%. However, the leakage of calcein caused by N151A, T152A, F157A, L183A, L185A and I188A were much lower than wild-type Cry2Ab, their maximum calcein release percentage were $24.33 \%, 20.72 \%, 35.98 \%, 32.36 \%, 37.53 \%$ and $32.53 \%$. Those data indicated that non-oligomerization mutants may weaken the pore-forming activities on liposome. Taken together, our data strongly suggested that oligomerization was tightly linked with the pore-forming activity of Cry2Ab.

\section{Discussion}


The helices $\mathrm{a}-4$ and $\mathrm{a}-5$ of Cry $1 \mathrm{~A}$ had been wildly reported as a pore-forming region (Girard et al. 2008; Torres et al. 2008). In other Cry toxin, helices $a-4$ and $a-5$ also reported in the oligomerization activity. Kanintronkul et al. reported that N166 and $\mathrm{Y} 170$ in helices a4-a5 was involved in the aggregation of Cry4B (Kanintronkul et al. 2003). Pornwiroon et al. reported that $Y 202$ in helices a4 was responded to the oligomerization activity of Cry4A (Pornwiroon et al. 2004). In our previous study also suggested that helices a4-a5 participate in Cry2Ab self-aggregation (Xu et al., 2018). In this study, we further constructed 20 alanine mutants site directed on helices a4-a5 and showed that residues N151, T152, F157, L183, L185 and I188 may be key residues for Cry2Ab oligomerization and insecticidal activity.

The pore-formation of Cry toxins on the membrane was one of the less characterized steps and was indispensable to fully understand the mechanism action of Cry toxins (Muñoz-Garay et al. 2006). Zavala et al. demonstrated that only Domain I inserted into the liposome while Domain II and III remained in the surface of membrane (Zavala et al. 2011). This result was consistence with the umbrella model of toxin insertion. It also revealed that residues V171 and T122 involved in the insertion of Cry1A toxin into members by fluorescent studies. Angsuthanasombat et al. revealed that residue Arg-136 participated in the membrane channel formation of Cry4Ba (Angsuthanasombat et al. 2001). It was also reported that residue N183 located in the middle of helix a-5 was crucial for the insecticidal activity and pore-forming activity of Cry4Ba (Likitvivatanavong et al. 2006). Similarly, our data showed that mutations on helice a-4 and a-5 (N151A, T152A, F157A, L183A, L185A and I188A) also blocked the pore-formation of Cry2Ab on liposome.

It was worth mentioning that Cry2Ab mutants which failed to assemble $250 \mathrm{kDa}$ oligomers, not only disrupted the insecticidal activity against $P$. xylostella, but also lost pore-forming activity of liposome. The results suggested that oligomerization and pore-formation was closely related in Cry2Ab. This mode of action was quite different from that of Cry $1 \mathrm{~A}$ toxin. For example, helices $\mathrm{a}-3$ and $\mathrm{a}-6$ of Cry $1 \mathrm{~A}$ was reported involved in oligomerization while helices a-4 and a-5 participated in pore-formation (JiménezJuárez et al. 2013; Lin et al. 2014). Furthermore, in Cry1A toxin, it was reported that toxins oligomerization required a binding step to cadherin, which promoted to cleavage of helix a-1 (Soberón et al. 2007). However, our previous data revealed that Cry2Ab could oligomerize in vitro after proteolysis by PxMJ in the absence of the cadherin (Xu et al. 2018). This diversity suggested that the mode of action of Cry2Ab might different from Cry1A toxin in some details and more researches were needed to clarify the elaborate difference. In conclusion, our study comprehensively identified key residues for Cry2Ab for the first time and demonstrated that non-oligomerization mutants affected the insecticidal activity as well as the pore-forming activity of Cry2Ab. It highlighted that oligomerization was closely related to insecticidal activity and pore-forming activity of Cry2Ab, which could make a closer look on the mode of action of Cry2Ab toxins.

\section{Declarations}

\section{Availability of data and material}


The supplementary materials are available online.

\section{Acknowledgements}

Not applicable.

\section{Authors' contributions}

YZ provided conceptual framework and technical oversight on all experiments; ZP and LX conducted the experiments and analyzed the experimental data. $Y Z, Z P$ and $L X$ wrote the manuscript; $Q C$ and $B L$ revised the manuscript. All authors read and approved the final manuscript.

\section{Funding}

The present investigation was supported by the National Natural Science Foundation of China (31972335), the Extended Project of National Natural Science Foundation of Fujian Academy of Agricultural Sciences (GJYS209003) and the Cultivation Fund of Fujian Academy of Agricultural Sciences (AGP208-4).

\section{Ethics approval and consent to participate}

This article does not contain any studies with human participants or animals performed by any of the authors.

\section{Consent for publication}

All authors have provided consent for this publication.

\section{Competing interests}

All the authors declare that they do not have any conflict of interest.

\section{References}

1. Bravo A, Likitvivatanavong S, Gill SS (2011) Bacillus thuringiensis: A story of a successful bioinsecticide. Insect Biochem Molec 41: 423-431.

2. Schnepf E, Crickmore NV, Van Rie J, Lereclus D, Baum J, Feitelson J, Dean DH (1998) Bacillus thuringiensis and its pesticidal crystal proteins. Microbiol Mol Bio Rev 62: 775-806.

3. Pardo-Lopez L, Soberon M, Bravo A (2013) Bacillus thuringiensis insecticidal three-domain Cry toxins: mode of action, insect resistance and consequences for crop protection, FEMS Microbiol Rev 37: 3-22.

4. Bravo A, Gill SS, Soberón M (2007) Mode of action of Bacillus thuringiensis Cry and Cyt toxins and their potential for insect control, Toxicon 49: 423-435. 
5. Vachon V, Laprade R, Schwartz JL (2012) Current models of the mode of action of Bacillus thuringiensis insecticidal crystal proteins: a critical review. J Invertebr Pathol 111: 1-12.

6. Pigott CR, Ellar DJ (2007) Role of receptors in Bacillus thuringiensis crystal toxin activity, Microbiol Mol Biol Rev 71: 255-281.

7. Bravo A, Gómez I, Porta H, García-Gómez Bl, Rodriguez-Almazan C, Pardo L, Soberón M (2013) Evolution of Bacillus thuringiensis Cry toxins insecticidal activity. Microb Biotechnol 6: 17-26.

8. Zhou Z, Liu Y, Liang G, Huang Y, Bravo A, Soberón M, Song F, Zhou X, Zhang J (2017) Insecticidal Specificity of Cry1Ah to Helicoverpa armigera is determined by binding of APN1 via domain II loops 2 and 3. Appl Environ Microb 83: e02864-16.

9. Park Y, Abdullah MAF, Taylor MD, Rahman K, Adang MJ (2009) Enhancement of Bacillus thuringiensis Cry3Aa and Cry3Bb toxicities to coleopteran larvae by a toxin-binding fragment of an insect cadherin. Appl Environ Microb 75: 3086-3092.

10. Guo Z, Kang S, Chen D, Wu Q, Wang S, Xie W, Zhang Y (2015) MAPK signaling pathway alters expression of midgut ALP and ABCC genes and causes resistance to Bacillus thuringiensis Cry $1 \mathrm{AC}$ toxin in diamondback moth. PLoS Genet 11: e1005124.

11. Chen J, Aimanova K, Gill SS (2017) Functional characterization of Aedes aegypti alkaline phosphatase ALP1 involved in the toxicity of Cry toxins from Bacillus thuringiensis subsp. israelensis and jegathesan. Peptides 98: 78-85.

12. Ferré J, Van Rie J (2002) Biochemistry and genetics of insect resistance to Bacillus thuringiensis. Annu Rev Entomol 47: 501-533.

13. Baxter SW, Badenes-Pérez FR, Morrison A, Vogel H, Crickmore N, Kain W, Jiggins CD (2011) Parallel evolution of Bt toxin resistance in Lepidoptera. Genetics 189: 675-679.

14. Xu L, Pan ZZ, Zhang J, Liu B, Zhu YJ, Chen QX (2016) Proteolytic activation of Bacillus thuringiensis Cry2Ab through a belt-and-braces approach. J Agr Food Chem 64: 7195-7200.

15. Liu C, Xiao Y, Li X, Oppert B, Tabashnik BE, Wu K (2014) Cis-mediated down-regulation of a trypsin gene associated with Bt resistance in cotton bollworm. Sci Rep 4: 7219.

16. Xia J, Guo Z, Yang Z, Zhu X, Kang S, Yang X, Xu W (2016) Proteomics-based identification of midgut proteins correlated with Cry1Ac resistance in Plutella xylostella (L.). Pestic Biochem Phys 132: 108117.

17. Jiménez-Juárez N, Muñoz-Garay C, Gómez I, Saab-Rincon G, Damian-Almazo JY, Gill, SS, Bravo A (2013) Bacillus thuringiensis Cry1Ab mutants affecting oligomer formation are non-toxic to Manduca sexta larvae. J Biol Chem 288: 8560-8560.

18. Vachon V, Préfontaine G, Coux F, Rang C, Marceau L, Masson L, Laprade, R (2002) Role of helix 3 in pore formation by the Bacillus thuringiensis insecticidal toxin Cry1Aa. Biochemistry 41: 6178-6184.

19. Soberón M, Pardo-López L, López I, Gómez I, Tabashnik BE, Bravo A (2007) Engineering modified Bt toxins to counter insect resistance. Science 318: 1640-1642. 
20. Muñoz-Garay C, Rodríguez-Almazán C, Aguilar JN, Portugal L, Gómez I, Saab-Rincon G, Bravo A (2009) Oligomerization of Cry11Aa from Bacillus thuringiensis has an important role in toxicity against Aedes aegypti. Appl Environ Microb 75: 7548-7550.

21. Pacheco S, Gómez I, Sánchez J, García-Gómez BI, Soberón M, Bravo A (2017) An intramolecular salt bridge in Bacillus thuringiensis Cry4Ba toxin is involved in the stability of helix a-3, which is needed for oligomerization and insecticidal activity. Appl Environ Microb 83: e01515-17.

22. Girard F, Vachon V, Préfontaine G, Marceau L, Su Y, Larouche G, Laprade R (2008) Cysteine scanning mutagenesis of a4, a putative pore-lining helix of the Bacillus thuringiensis insecticidal toxin Cry $1 \mathrm{Aa}$. Appl Environ Microbiol 75: 2565-2572.

23. Torres J, Lin X, Boonserm P (2008) A trimeric building block model for Cry toxins in vitro ion channel formation. Biochim Biophys Acta 1778: 392-397.

24. Xu L, Pan ZZ, Zhang J, Niu LY, Li J, Chen Z, Zhu YJ, Chen QX (2018) Exposure of helices a4 and a5 is required for insecticidal activity of Cry2Ab by promoting assembly of a prepore oligomeric structure. Cell Microbiol 20: e12827.

25. Pan ZZ, Xu L, Zhu YJ, Shi H, Chen Z, Chen M, Chen QX, Liu B (2014) Characterization of a new cry2Ab gene of Bacillus thuringiensis with high insecticidal activity against Plutella xylostella $\mathrm{L}$.. World J Microb Biot 30: 2655-2662.

26. Finney DJ (1971) Probit Analysis 3rd edition. Cambridge University Press London.

27. Ding J, Wang K, Liu W, She Y, Sun Q, Shi J, Shao F (2016) Pore-forming activity and structural autoinhibition of the gasdermin family. Nature 535: 111.

28. Kanintronkul Y, Sramala I, Katzenmeier G, Panyim S, Angsuthanasombat C (2003) Specific mutations within the a4-a5 loop of the Bacillus thuringiensis Cry4B toxin reveal a crucial role for Asn-166 and Tyr-170. Biochem Biophys Res Commun 24: 11-19.

29. Pornwiroon W, Katzenmeier G, Panyim S, Angsuthanasombat C (2004) Aromaticity of Tyr-202 in the a45 loop is essential for toxicity of the Bacillus thuringiensis Cry4A toxin. J Biochem Mol Biol 37: 292-297.

30. Muñoz-Garay C, Sánchez J, Darszon A, De Maagd RA, Bakker P, Soberón M, Bravo A (2006) Permeability changes of Manduca sexta midgut brush border membranes induced by oligomeric structures of different Cry toxins. J Membrane Biol 212: 61-68.

31. Zavala LE, Pardo-López L, Cantón PE, Gómez I, Soberón M, Bravo A (2011) Domains II and III of Bacillus thuringiensis Cry $1 \mathrm{Ab}$ toxin remain exposed to the solvent after insertion of part domain I into the membrane. J Biol Chem 286: 19109-19117.

32. Angsuthanasombat C, Keeratichamreon S, Leetacheewa S, Katzenmeier G, Panyim S (2001) Directed mutagenesis of the Bacillus thuringiensis Cry $11 \mathrm{~A}$ toxin reveals a crucial role in larvicidal activity of arginine-136 in helix 4. BMB Rep 34: 402-407.

33. Likitvivatanavong S, Katzenmeier G, Angsuthanasombat C (2006) Asn183 in a5 is essential for oligomerisation and toxicity of the Bacillus thuringiensis Cry4Ba toxin. Arc Biochem Biophys 445: 4655 . 
34. Lin X, Parthasarathy K, Surya W, Zhang T, Mu Y, Torres J (2014) A conserved tetrameric interaction of cry toxin helix a3 suggests a functional role for toxin oligomerization. BBA - Biomembranes 1838: 1777-1784.

\section{Figures}
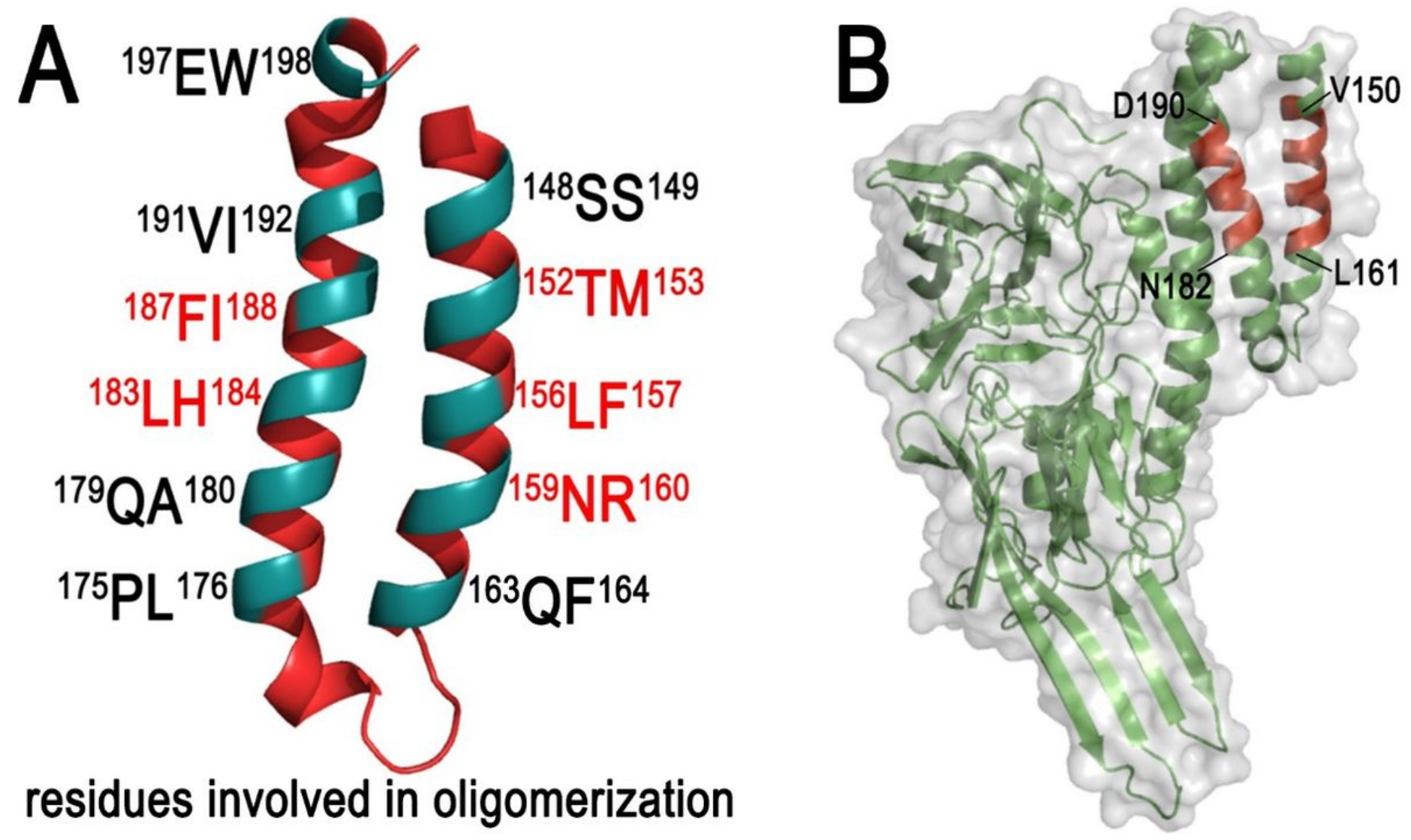

Figure 1

(A) Helices a4-a5 were involved in Cry2Ab oligomerization. (B)The design and construction of alanine mutants of Cry2Ab site-directed within the helices a4-a5. 

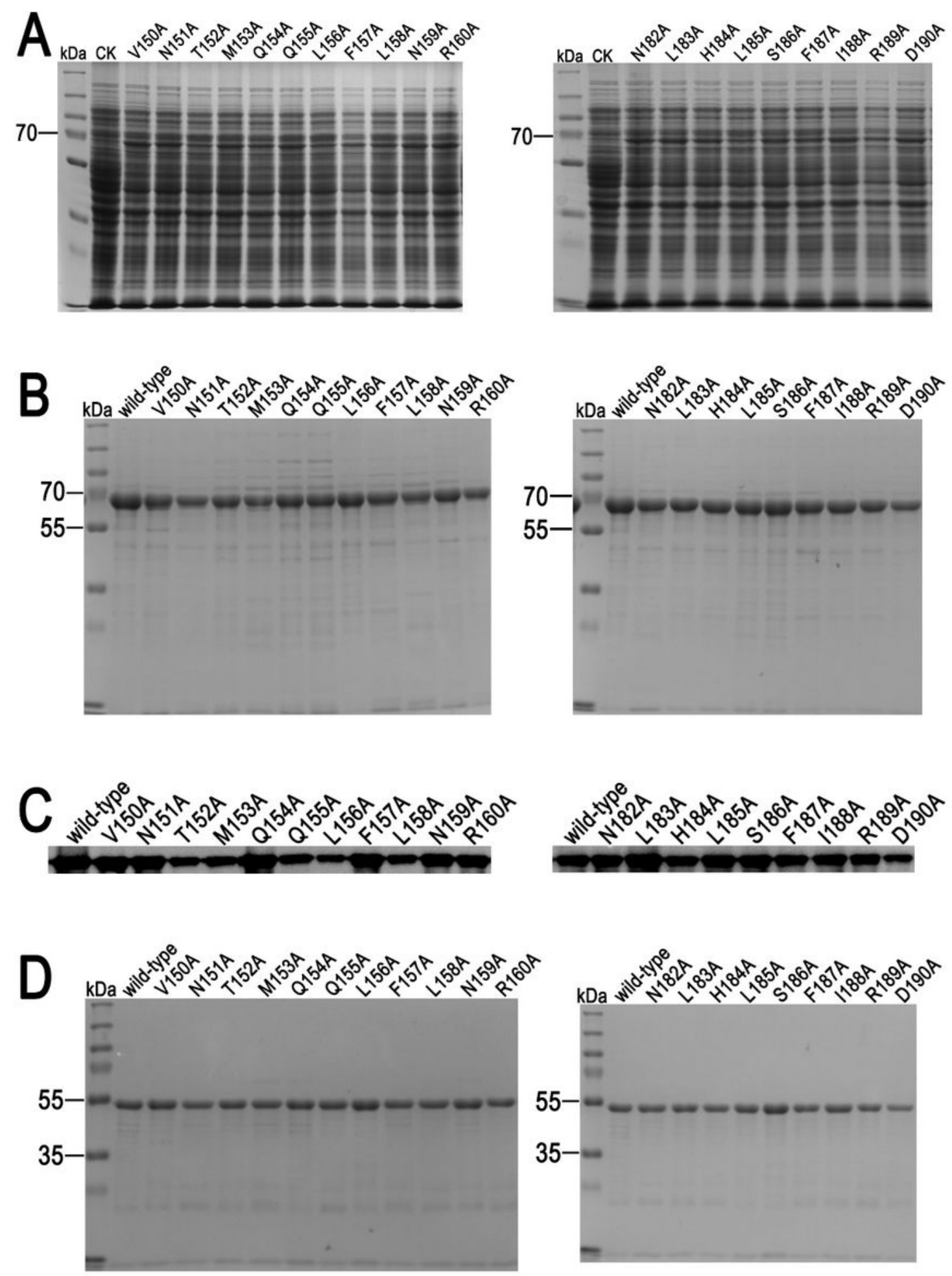

\section{Figure 2}

Expression, purification and identification of Cry2Ab mutants. (A)The induced expression of Cry2Ab variants site-directed on helices a4-a5. (B) The purity of Cry2Ab mutants detected by SDS-PAGE followed by Coomassie blue staining. (C) Identification of Cry2Ab mutants using an anti-Cry2Ab antibody. (D) The proteolytic activation of Cry2Ab mutants by PxMJ. 


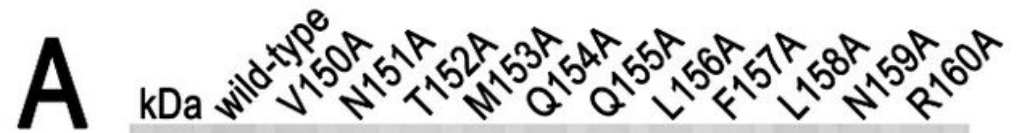

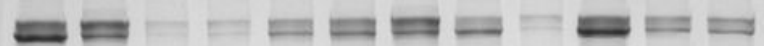
250

$-$
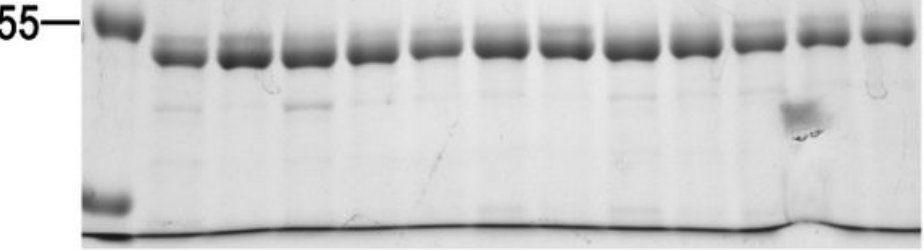

B

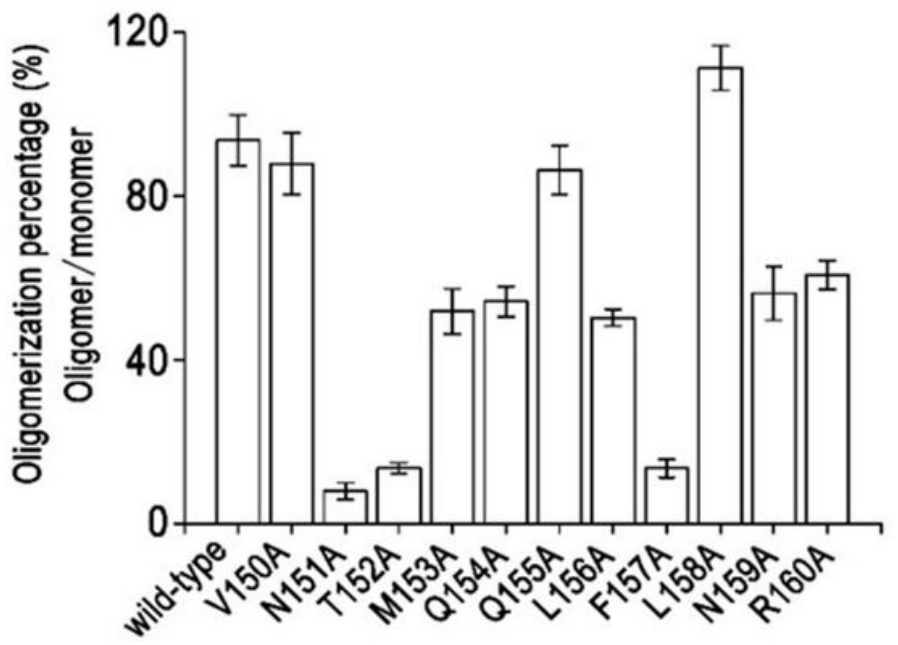

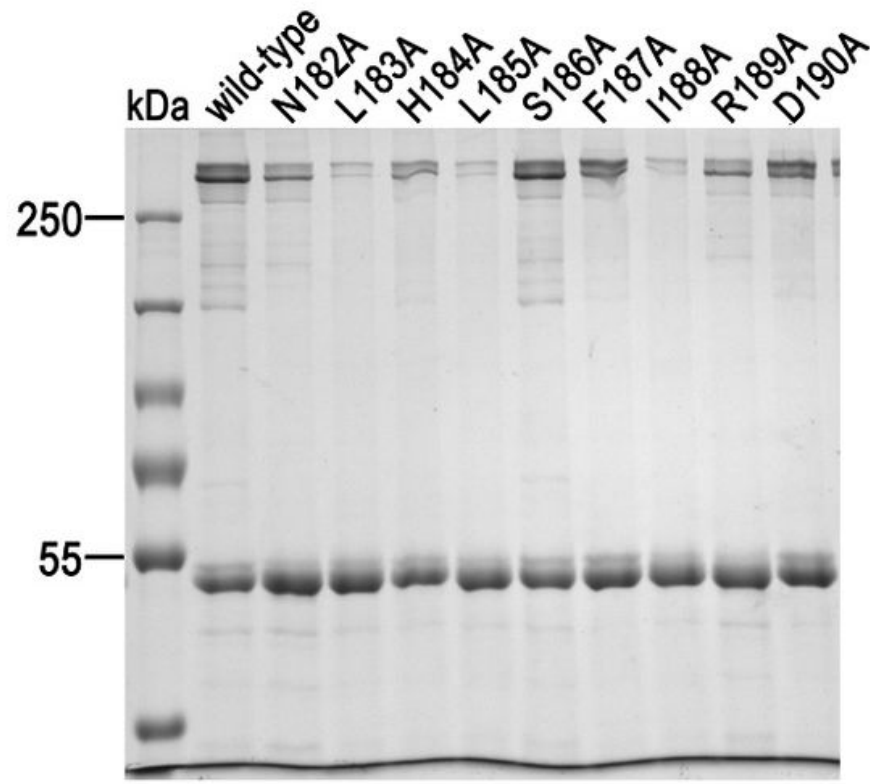

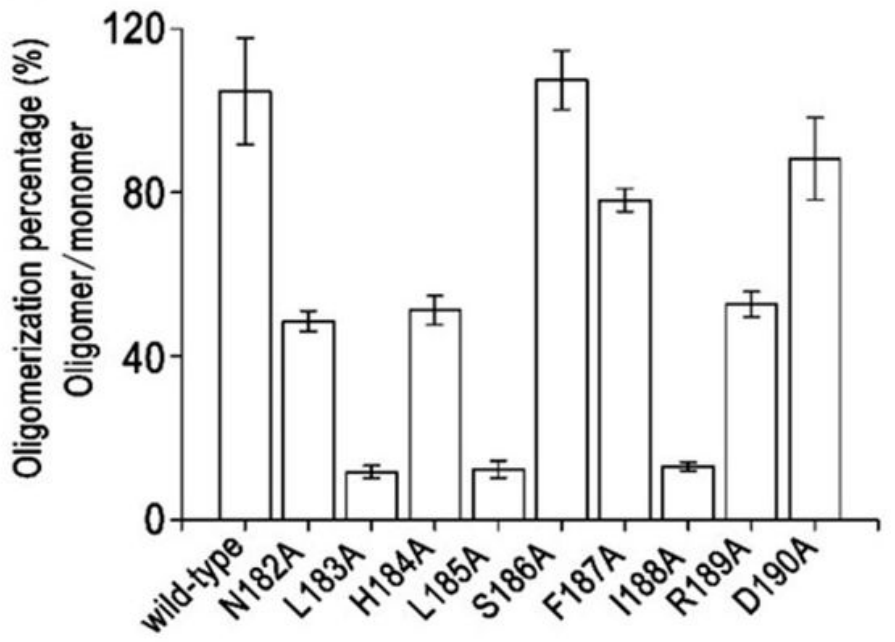

Figure 3

Oligomerization assay of Cry2Ab mutants. (A) Assemble of Cry2Ab oligomers detected by SDS-PAGE followed by Coomassie blue staining. (B) Calculation of the percentage of oligomer and monomer in Cry2Ab by image $\mathrm{J}$. 


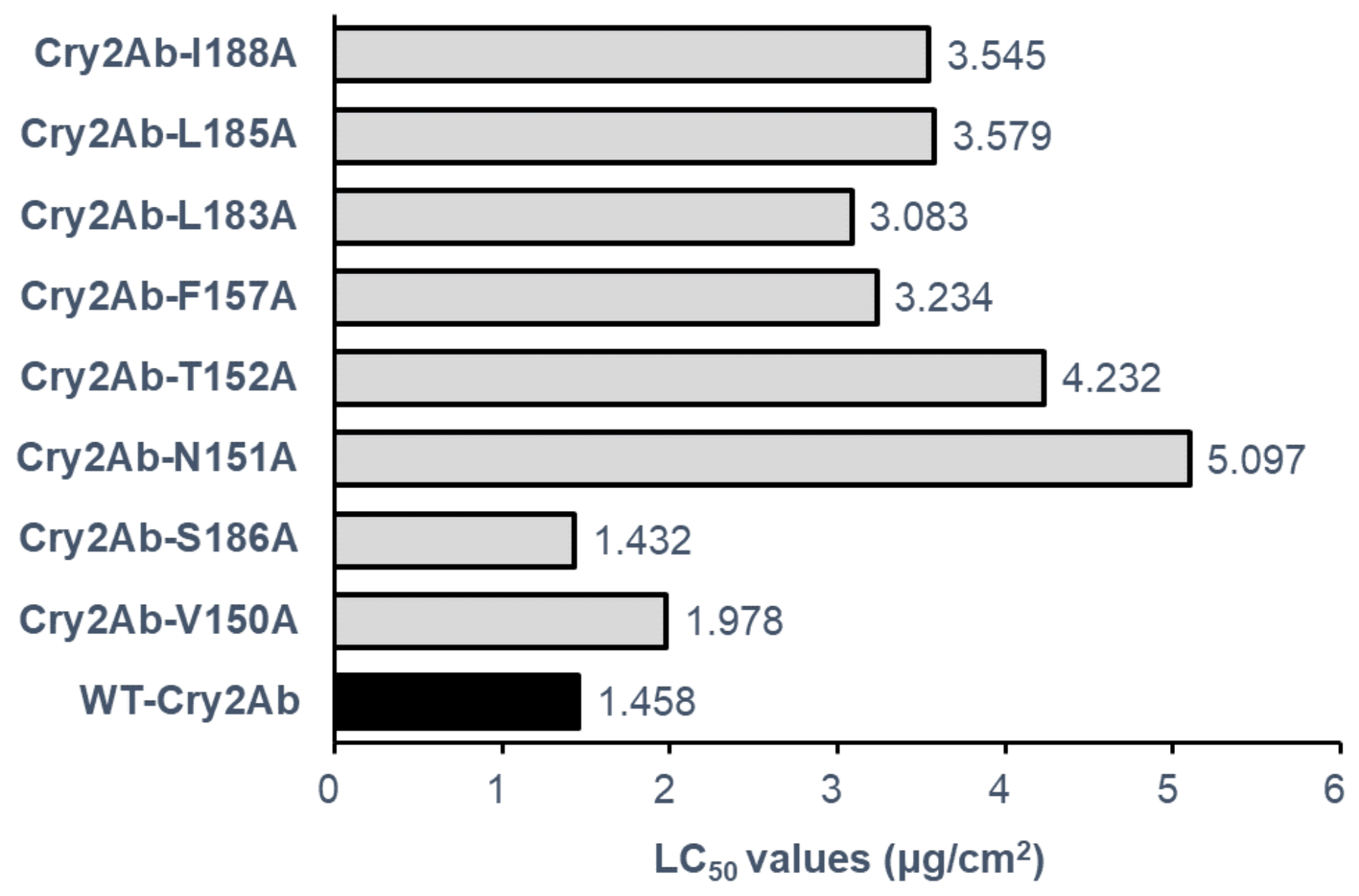

Figure 4

Comparison LC50 values of mutants Cry2Ab (site-directed on the helices a4-a5) against 2nd instar larvae of Plutella xylostella with wild-type Cry2Ab. WT-Cry2Ab means the wild-type Cry2Ab. 

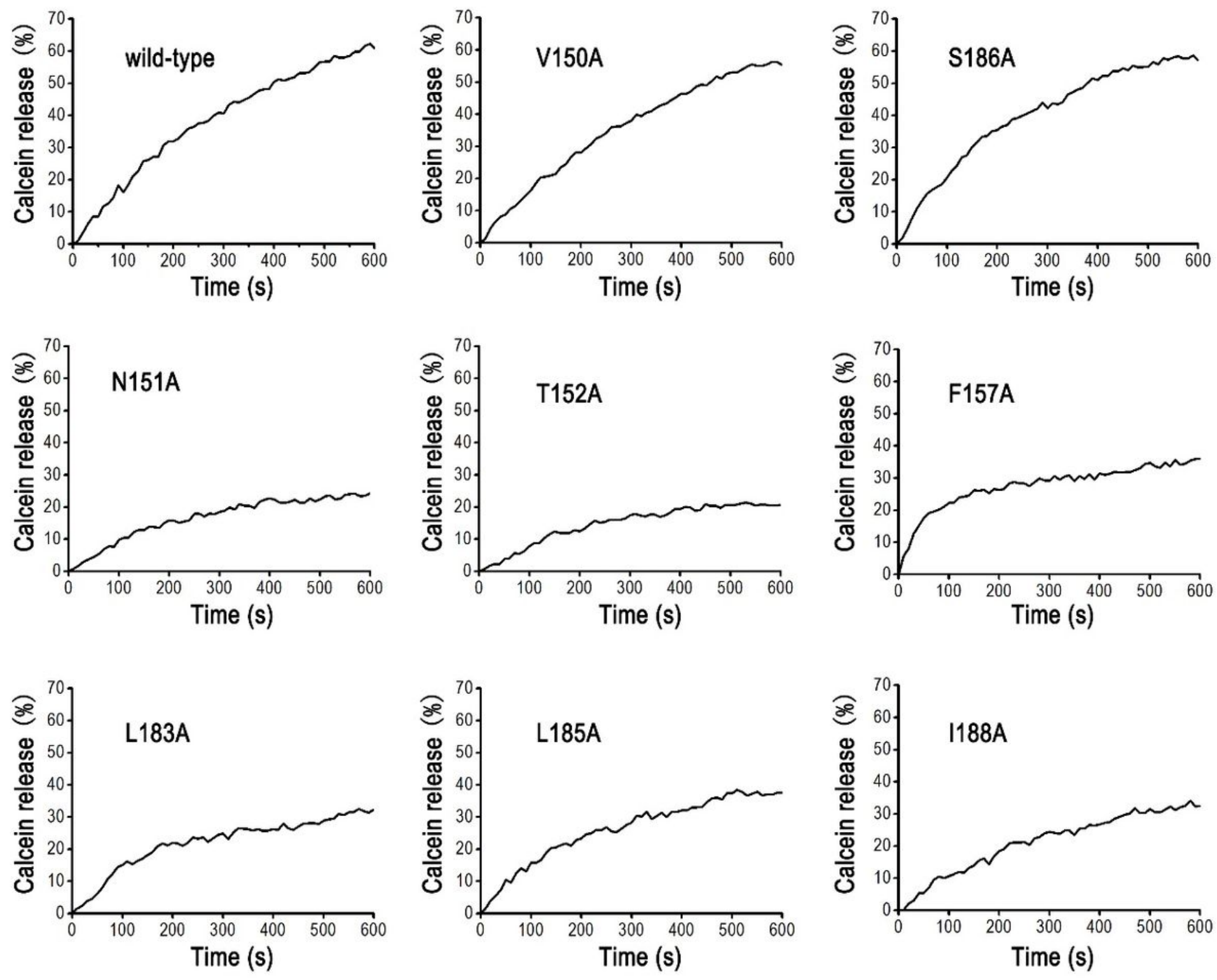

Figure 5

Liposome leakage assay detected pore-forming activity of Cry2Ab mutants.

\section{Supplementary Files}

This is a list of supplementary files associated with this preprint. Click to download.

- SupplementaryMaterial.docx 